\title{
Validation of a new serological assay for the identification of Mycobacterium tuberculosis complex-specific antibodies in pigs and wild boar
}

\author{
Jobin Thomas ${ }^{\mathrm{a}, \mathrm{b}}$, Jose A. Infantes-Lorenzo ${ }^{\mathrm{c}, \mathrm{d}}$, Inmaculada Moreno ${ }^{\mathrm{e}}$, David Cano-Terriza ${ }^{\mathrm{f}}$, \\ Lucía de Juan $^{\mathrm{c}, \mathrm{d}}$, Ignacio García-Bocanegra ${ }^{\mathrm{f}}$, Lucas Domínguez ${ }^{\mathrm{c}, \mathrm{d}}$, Mercedes Domínguez ${ }^{\mathrm{e}}$, \\ Christian Gortázar ${ }^{\mathrm{a}, *}$, María A. Risalde ${ }^{g, h}$ \\ ${ }^{a}$ SaBio (Health and Biotechnology), Instituto de Investigación en Recursos Cinegéticos IREC (CSIC-UCLM), Ciudad Real, Spain \\ ${ }^{\mathrm{b}}$ Indian Council of Agricultural Research (ICAR), New Delhi, India \\ ${ }^{\mathrm{c}}$ VISAVET Health Surveillance Centre, Complutense University of Madrid, Madrid, Spain \\ ${ }^{\mathrm{d}}$ Dpto. de Sanidad Animal, Facultad de Veterinaria, Universidad Complutense, Madrid, Spain \\ ${ }^{\text {e } U n i d a d ~ d e ~ I n m u n o l o g i ́ a ~ M i c r o b i a n a, ~ C e n t r o ~ N a c i o n a l ~ d e ~ M i c r o b i o l o g i ́ a, ~ I n s t i t u t o ~ d e ~ S a l u d ~ C a r l o s ~ I I I, ~ M a j a d a h o n d a, ~ M a d r i d, ~ S p a i n ~}$ \\ ${ }^{\mathrm{f}}$ Dpto. de Sanidad Animal, Facultad de Veterinaria, Universidad de Córdoba (UCO), Agrifood Excellence International Campus (ceiA3), Córdoba, Spain \\ ${ }^{g}$ Dpto. de Anatomía y Anatomía Patológica Comparadas, Facultad de Veterinaria, Universidad de Córdoba (UCO), Agrifood Excellence International Campus (ceiA3), \\ Córdoba, Spain \\ ${ }^{\mathrm{h}}$ Infectious Diseases Unit. Instituto Maimonides de Investigación Biomédica de Córdoba (IMIBIC). Hospital Universitario Reina Sofía de Córdoba. Universidad de Córdoba. \\ Cordoba, Spain
}

\section{A R T I C L E I N F O}

Keywords:

ELISA assay

In vivo diagnosis

Mycobacterium tuberculosis complex

Sensitivity

Specificity

\begin{abstract}
A B S T R A C T
Background and objectives: Animal tuberculosis (TB) is a multi-host disease involving a wide variety of domestic and wild mammals and causing a significant economic burden and sanitary problems. Wild boar and domestic pigs (Sus scrofa) are indicators of the circulation of the Mycobacterium tuberculosis complex (MTC) and can play a role in its maintenance. The proper diagnosis of MTC contact in these species is, therefore, a key factor as regards controlling TB. The objective of the current study is to evaluate the diagnostic performance of the protein complex P22 as a candidate for use in an in-house ELISA to identify $M$. tuberculosis complex-specific antibodies for the diagnosis of TB in comparison to the commonly used bPPD-based ELISA (bPPD ELISA) in suids.

Methods: We conducted a retrospective study. Sera were collected from wild boar during hunting season and from domestic pigs during routine handling, and all the animals underwent reference standard tests (detailed necropsy followed by bacteriological culture and isolation). Animal TB was confirmed to be positive in 277 animals and negative in 366 animals based on both reference standard tests. Sera from those animals were tested by P22 ELISA as well as bPPD ELISA.

Results: Both ELISAs yielded a good diagnostic value, however, a higher sensitivity (Se) and specificity (Sp) was achieved with the P22 ELISA (Se: 84.1\%; $\mathrm{CI}_{95 \%}$ : 79.3-88.2\% / Sp: 98.4\%; $\mathrm{CI}_{95 \%}: 96.5-99.4 \%$ ) when compared to the bPPD ELISA (Se: 77.3\%; $\mathrm{CI}_{95 \%}$ : 71.9-82.2\% / Sp: 97.3\%; $\mathrm{CI}_{95 \%}$ : 95-98.3\%). An optimum Sp of $100 \%$ (CI $_{95 \%}$ : 98.54-100\%) was attained with white pigs for both the bPPD and the P22 ELISA.

Discussion: The results suggest that serological tests for MTC-antibody detection, and particularly the P22 ELISA, are valuable tools in the diagnosis of TB in wild boar and domestic pigs when attempting to detect contact with MTC and thereby facilitate TB control and management.
\end{abstract}

\section{Introduction}

Animal tuberculosis (TB) is a globally relevant multi-host disease caused by Mycobacterium bovis and other closely related members of the M. tuberculosis complex (MTC), which infect a wide variety of mammals including domestic and wild animals (Waters et al., 2016). This disease causes a significant economic burden and sanitary problems in the farming industry (Chambers, 2013), and is also responsible for zoonotic problems, mainly in developing countries (Michel et al., 2010).

The role of wild and domestic hosts in the epidemiology of animal TB varies between regions (Gortázar et al., 2012). Suids have been recognized to play a significant epidemiological role in animal TB in

\footnotetext{
* Corresponding author.

E-mail address: Christian.Gortazar@uclm.es (C. Gortázar).
} 
different areas, particularly in the case of the Eurasian wild boar (Sus scrofa). Wild boar TB has been reported in many European countries (Gortázar et al., 2015), in addition to Asia (Jang et al., 2017), northern Africa (El Mrini et al., 2016), and South America (Maciel et al., 2018). Moreover, scientific evidence suggesting that this species plays a key role in the maintenance of MTC on the Iberian Peninsula is accumulating (Naranjo et al., 2008; Gortázar et al., 2015, 2017).

Domestic pigs (and feral swine) are often considered as 'dead end hosts' for MTC (Corner, 2006; Nugent et al., 2015). However, in certain epidemiological scenarios, several studies have shown that free-ranging domestic pigs can act as maintenance hosts for MTC, becoming a true reservoir (Di Marco et al., 2012) or amplifier host (Coleman and Cooke, 2001). In any case, domestic pigs and feral swine are useful sentinels for MTC (Corner, 2006; Bailey et al., 2013; Nugent et al., 2015). Animal TB has also been reported in other suid species such as bush pigs (Potamochoerus porcus) (Hlokwe et al., 2014) and warthogs (Phacochoerus africanus) in Africa (Miller et al., 2016) and collared peccaries (Tayassu tajacu) in South America (Mayer et al., 2012).

Hence, proper identification of wild and domestic suids infected with $\mathrm{TB}$, followed by implementation of suitable management strategies, is important for an effective TB control (Csivincsik et al., 2016). Standard in vivo diagnostic methods included in the current eradication programs of animal TB, generally in cattle and other ruminants, are based on measuring the cell-mediated immune (CMI) response to MTC (Buddle et al., 2009), which can be detected by using the tuberculin skin test or interferon-gamma (IFN $\gamma$ ) release assays (De la RuaDomenech et al., 2006). Similar CMI-based tests are also available for suids, but have logistic constraints and are therefore not the first choice for these species (Jaroso et al., 2010; Pesciaroli et al., 2012; Beltrán-Beck et al., 2014).

Antibody-based diagnosis has been widely used on wild boar and feral swine owing to its ease of use and generally good performance (Boadella et al., 2011; Richomme et al., 2010; Pedersen et al., 2017). Table 1 presents the main serological assays used on wild boar and in pigs/feral swine. The most commonly used antigen is the purified protein derivative obtained from $M$. bovis (bovine PPD or bPPD) (Aurtenetxe et al., 2008; Boadella et al., 2011; Che'Amat et al., 2015); however, recent studies have focused on improving its specificity (Sp) by using more specific antigens, such as MPB83 (García-Bocanegra et al., 2012; Pérez de Val et al., 2017), MPB70 (Cardoso-Toset et al., 2017) or CFP10/ESAT-6 (Roos et al., 2016). New ELISA assays recently performed with an immunopurified subcomplex protein obtained from bPPD (CZ Veterinaria SA, Porriño, Spain), named P22 and composed of several antigens including MPB70, MPB83, ESAT-6 and CFP-10 (Infantes-Lorenzo et al., 2017), have provided a high Sp and sensitivity (Se) in cattle and mice (Casal et al., 2017; Infantes-Lorenzo et al., 2017). Therefore, we hypothesized that P22 ELISA would yield a higher diagnostic performance than bPPD ELISA in terms of Se and Sp for TB diagnosis in suids.

The present study was conducted with the objective of evaluating the diagnostic performance of P22 as a candidate for use in a new serological assay with which to identify $M$. tuberculosis complex-specific antibodies for the diagnosis of TB in wild boar and domestic pig.

\section{Materials and methods}

\subsection{Animals}

The study was performed retrospectively with samples from wild boar and domestic pigs collected from several sources. We included samples from wild boar, domestic pig in extensive management system (Iberian pig), as well as domestic pig in intensive management system (white pig) in order to avoid bias in the validation of the test and also to assess the performance of the test in two different systems of management, because of the fact that animals reared under extensive management systems or wildlife have more chances of exposure to MTC and other environmental mycobacteria, compared to the animals reared under intensive management systems. The animals were not intentionally killed for this study but were rather hunter-harvested (wild boar) during the legal hunting season 2015-16 from September to March or routinely slaughtered (pigs) during the period 2015-16 from the southern and central Spain. No ethical approval was, therefore, deemed necessary for this study. Protocols, amendments and other resources were used according to the guidelines approved by each autonomous government following the R.D.1201/2005 of the Spanish Ministry of Presidency. Blood and tissue samples were collected by veterinarians in compliance with the Ethical Principles in Animal Research. The study was compiled in accordance with STARD guidelines 2015 (Cohen et al., 2016).

All animals were subjected to a detailed post-mortem examination in order to assess the presence or absence of TB compatible lesions (TBL). Samples included in the study were selected using a consecutive sampling technique and sample size for index test validation was determined by incorporating all the samples from wild boar and domestic pigs with confirmed infection status (both positive and negative). The infection status was assessed on the basis of the presence of TBL followed by bacteriological culture and isolation (reference standard test). In this study, a TB-positive animal is defined as the one that has TBL and is also positive to the MTC culture, while a TB-negative animal is negative as regards both TBL and the MTC culture. The reference standard tests were performed in another laboratory and the results of reference standard were available to the assessors of index test and vice versa. Details of animals used for index test validation are summarized in Table 2.

\subsection{Bacteriology}

Tissue samples were placed in a sterile recipient and stored at $-80^{\circ} \mathrm{C}$ until their bacteriological culture. All tissues with present or suspicious TBLs were cultured for MTC. When TBLs were not observed, a pool of mandibular, tracheobronchial and mediastinal lymph nodes (LNs) from each animal was also cultured. The culture of samples was performed on Coletsos and $0.2 \%(\mathrm{w} / \mathrm{v})$ pyruvate-enriched LöwensteinJensen media (Difco, Madrid, Spain) after decontamination with a final concentration of $0.37 \%$ hexadecylpyridinium chloride (Corner and Trajstman, 1988). Isolates were identified by means of PCR (Wilton and Cousins, 1992) and spoligotyping (Kamerbeek et al., 1997).

\subsection{Serum antibody detection}

Blood samples (approximately $10 \mathrm{ml}$ from each animal) were collected in tubes without additives by puncture of the orbital sinus in pigs and the cavernous sinus of the dura mater in wild boar (Arenas-Montes et al., 2013). Samples were centrifuged ( $1500 \mathrm{~g}$ for $10 \mathrm{~min}$ ) and sera stored at $-20^{\circ} \mathrm{C}$ until testing for the detection of MTC antibodies.

\subsubsection{Indirect ELISA with $P 22$}

Serum samples were tested by means of an in-house ELISA using P22 as antigen and following the protocol described by Casal et al. (2017) with some modifications. Briefly, after coating the plates with the P22 antigen at a concentration of $10 \mu \mathrm{g} / \mathrm{ml}$ in phosphate buffered saline (PBS) (ingredients of PBS; Panreac Química S.L.U., Barcelona, Spain), they were stored overnight at $4{ }^{\circ} \mathrm{C}$. The wells were subsequently washed with a PBS solution containing $0.05 \%$ Tween-20 (PBST) (Tween 20; Sigma Aldrich Inc., Munich, Germany) and blocked for $1 \mathrm{~h}$ at room temperature (RT) with $5 \%$ skimmed milk powder (commercially available) solution in PBS (SM). Sera were added in duplicate at a dilution of $1: 100$ in SM, incubated stationary for $1 \mathrm{~h}$ at $37^{\circ} \mathrm{C}$ and subsequently washed three times with PBST. Anti-porcine IgG (HRP)conjugated (Bethyl Laboratories, Montgomery, USA) at a concentration of 1:20,000 in SM was added and the plates were incubated for $1 \mathrm{~h}$ at RT. Following four washes with PBST, color was developed with o- 


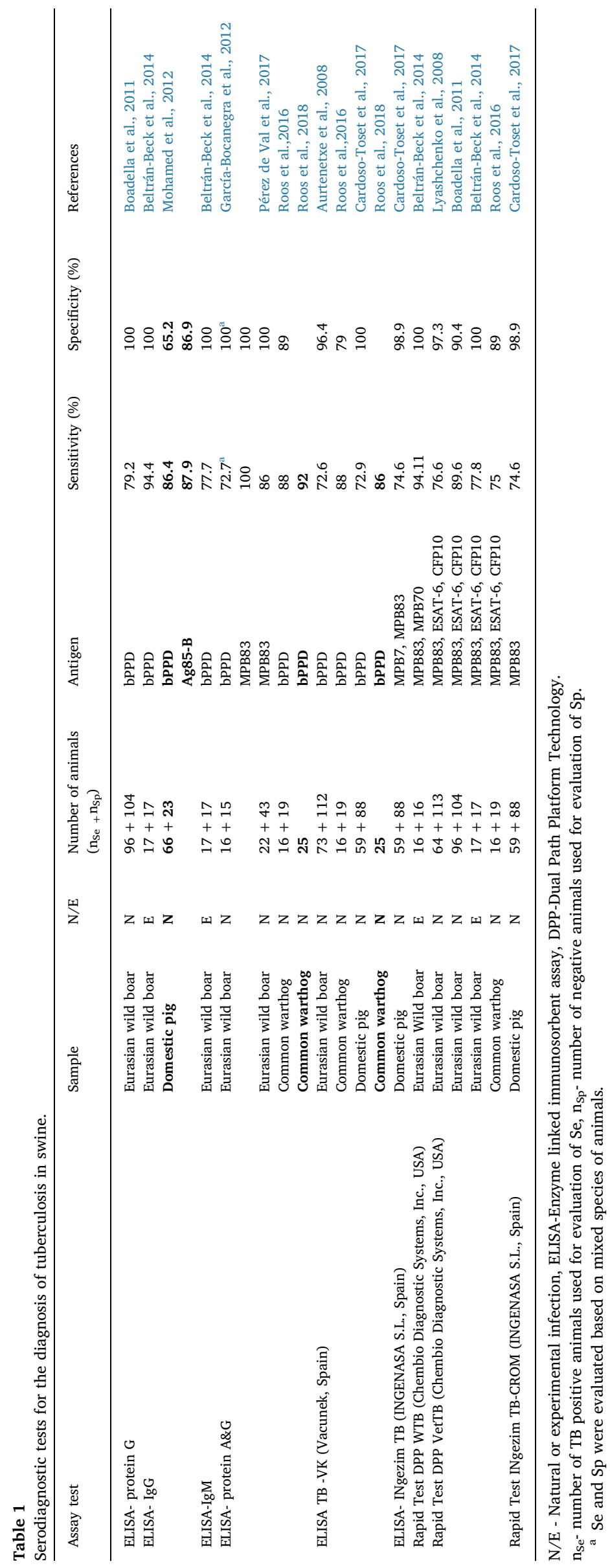


Table 2

Details of the animals used for validation of index test.

\begin{tabular}{lll}
\hline Sample & Number & TB status $^{\mathrm{a}}$ \\
\hline Wild boar & 236 & TB-positive \\
& 103 & TB-negative \\
Iberian pig & 41 & TB-positive \\
& 12 & TB-negative \\
White pig & 251 & TB-negative \\
Total = 643 (277 TB-positive and 366 TB-negative animals) &
\end{tabular}

a TB-positive: presence of TB-compatible lesions and positive MTC culture; TB-negative: absence of TB-compatible lesions and negative MTC culture.

phenylenediamine dihydrochloride substrate (FAST OPD, Sigma Aldrich Inc., Munich, Germany), and the samples were incubated for 20 min in darkness and RT conditions. The reaction was stopped with $\mathrm{H}_{2} \mathrm{SO}_{4} 2 \mathrm{M}$ and the optical density (OD) measured in a spectrophotometer at $450 \mathrm{~nm}$.

With the aim of evaluating the repeatability of the assay, the same positive and negative controls were included in quadruplicate in every plate of the study (which were tested on the same day and on different days, as well as by different operators). The positive controls were obtained from MTC infected wild boar, previously confirmed by the presence of TBL and M. bovis culture, as well as by bPPD ELISA (high and low ODs) for MTC-antibodies. The negative controls were obtained from TB-free wild boar from TB-free areas, previously confirmed as MTC negative by culture and without the presence of TBL, and negative to bPPD ELISA for MTC-antibodies.

\subsubsection{Indirect ELISA with $b P P D$}

The serum samples were tested by following the same in-house ELISA protocol described above, but using bPPD (CZ Veterinaria SA, Porriño, Spain) as antigen at a concentration of $5 \mu \mathrm{g} / \mathrm{ml}$. The positive and negative controls used were the same as for P22 antigen. This methodology, with slight modifications, has been previously used for the identification of MTC specific antibodies in wild boar and domestic pigs (Che'Amat et al., 2015; Cardoso-Toset et al., 2017).

\subsection{Data analysis}

The ELISA results were expressed as an ELISA percentage (E\%), calculated using the following formula: [sample $\mathrm{E} \%$ = (mean sample $\mathrm{OD} /(2 \mathrm{x}$ mean of negative control OD $)) \times 100]$. The cut-off value was calculated using a ROC analysis and was defined as the value at which the highest sum of Se plus Sp was obtained (Aurtenetxe et al., 2008). The presence or absence of TBL and mycobacterial culture was employed as a reference standard. The discriminatory indexes for Se and Sp were calculated as the ratio between each one and the complementary indexes of the other (Se/(1-Sp) and $\mathrm{Sp} /(1-\mathrm{Se})$, respectively) in order to assess test performance. Confidence intervals for the positive and negative predictive values (PPV and NPV, respectively) of the Se and $\mathrm{Sp}$ were calculated using the Clopper-Pearson method (Graphpad prism 5.0, USA). The inter-test agreement between the evaluated assays was calculated by means of Cohen's kappa coefficient (Epitools, Ausvet Pty Ltd., Canberra, Australia). All statistical analyses were performed at a confidence level of $95 \%\left(\mathrm{CI}_{95 \%}\right)$.

\section{Results}

The presence of TBL was diagnosed by necropsy of the entire animal with macroscopic inspection of LNs, abdominal and thoracic organs, where lesions were especially prominent in lung, as well as in mandibular and tracheobronchial LNs. The presence of TBL was confirmed or not by isolation of the microorganism through MTC culture. Animals found to be positive to either TBL only or culture only were omitted from the study. Animals positive or negative to both techniques were

\section{P22 ELISA}

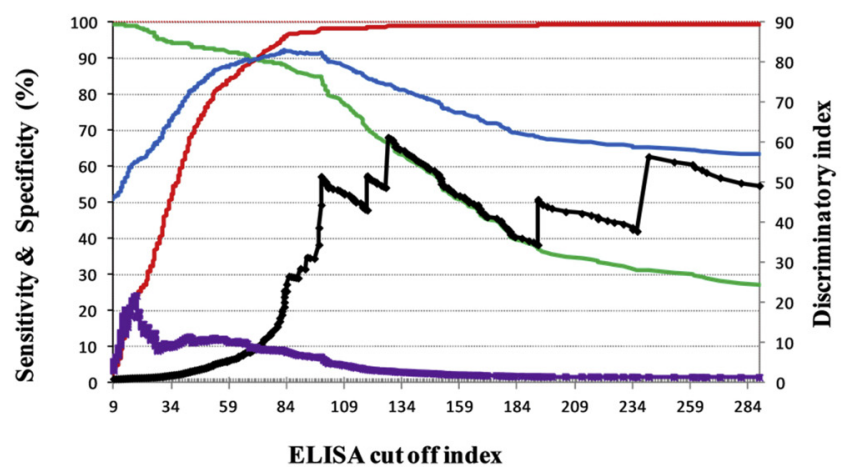

bPPD ELISA

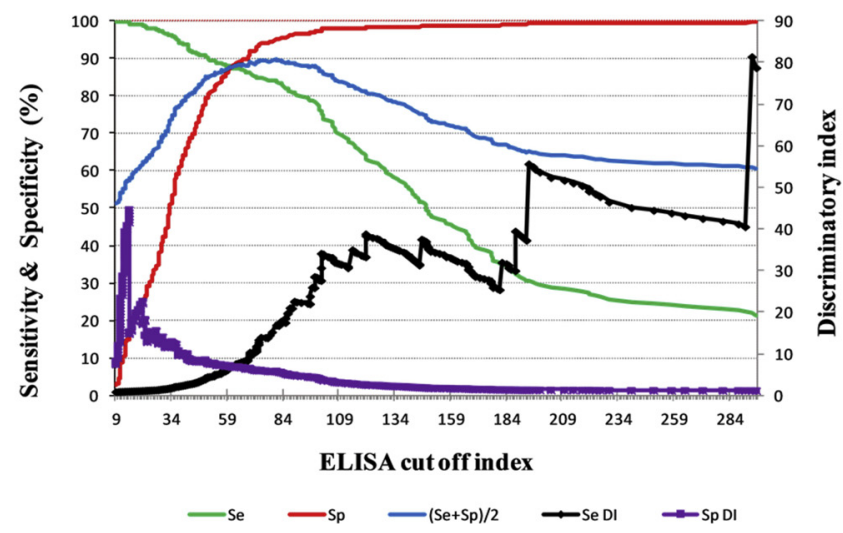

Fig. 1. Diagnostic value graphs for the serodiagnosis of tuberculosis in swine (wild boar and domestic pigs) when using the Mycobacterium bovis-derived immunopurified P22 or the purified protein derivative (bPPD) as antigens. Sensitivity (Se), specificity (Sp) and their semi-sum are the percentages on the primary Y-axis. Discriminatory indexes for Se [Se/ (1-Sp)] and Sp [(Sp/ (1-Se)] are on the secondary Y-axis.

included in the study for index test validation. The flow of participants used in this study is summarized in a diagram in Supplementary material (Fig. $\mathrm{S}^{-1}$ ).

The diagnostic value of P22 and bPPD ELISA in swine, obtained by means of a ROC analysis (Fig. 1), shows that the P22 ELISA had a slightly better sum of Se + Sp (184) than the bPPD ELISA (179). The cut-off value was defined as the ratio of the mean sample OD to the double of the mean OD of the negative control. The P22 ELISA with a cut-off value set at $100 \mathrm{E} \%$ had both a higher Se (84.1\%) and a higher Sp (98.4\%) when compared to the values attained with bPPD ELISA (Se: $77.3 \%$, Sp: $97.3 \%$ ). Modifying the cut-off value (greater than or less than $100 \mathrm{E} \%$ ) resulted in either a decreased Sp or a constant Se, so that a cut-off value of 100 was chosen for the P22 ELISA. Moreover, both discriminatory indices of Se and Sp reached their optimum value in P22 ELISA at the cut-off value chosen (100 E\%), being higher than those achieved in bPPD ELISA and indicating a good level of ability to predict and discriminate the true positive and true negative individuals. The cross tabulation of P22 and bPPD ELISA results by reference standard is summarized in Supplementary material (Table S-2).

Data regarding Se, Sp, PPV, NPV, area under the curve (AUC), and kappa values (K), along with a $\mathrm{CI}_{95}$ for both ELISAs at the chosen cut-off value of $100 \mathrm{E} \%$ are summarized in Table 3 . In this context, if Sp is evaluated within the different management systems, we observe that it was $94.78 \%\left(\mathrm{CI}_{95}: 88.99-98.06 \%\right)$ and $91.30 \%\left(\mathrm{CI}_{95}: 84.59-95.75 \%\right)$ for P22 and bPPD ELISA, respectively, in animals reared under extensive management systems (wild boar and Iberian pig). However, an optimum Sp of $100 \%\left(\mathrm{CI}_{95}: 98.54-100 \%\right)$ was attained with animals 
Table 3

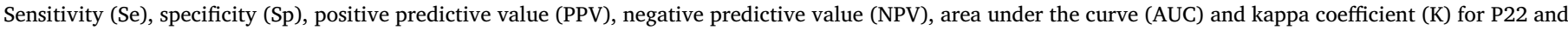
bPPD ELISA in swine.

\begin{tabular}{|c|c|c|c|c|c|c|c|c|c|c|c|c|}
\hline \multirow[t]{2}{*}{ Test } & \multicolumn{2}{|l|}{$\mathrm{Se}$} & \multicolumn{2}{|l|}{ Sp } & \multicolumn{2}{|l|}{ PPV } & \multicolumn{2}{|l|}{ NPV } & \multicolumn{2}{|l|}{ AUC } & \multicolumn{2}{|l|}{$\mathrm{K}$} \\
\hline & $\%$ & $\mathrm{Cl}_{95}^{\mathrm{a}}$ & $\%$ & $\mathrm{Cl}_{95}$ & $\%$ & $\mathrm{Cl}_{95}$ & $\%$ & $\mathrm{Cl}_{95}$ & & $\mathrm{Cl}_{95}$ & & $\mathrm{Cl}_{95}$ \\
\hline P22 ELISA & 84.1 & $79.3-88.2$ & 98.4 & $96.5-99.4$ & 97.5 & $94.6-99.1$ & 89.1 & $85.7-92.0$ & 0.94 & $0.92-0.96$ & 0.84 & $0.8-0.88$ \\
\hline $\begin{array}{l}\text { bPPD } \\
\text { ELISA }\end{array}$ & 77.3 & 71.9-82.1 & 97.3 & $95-98.3$ & 95.5 & $92-97.8$ & 85.0 & $81.2-88.3$ & 0.93 & $0.91-0.95$ & 0.76 & $0.71-0.81$ \\
\hline
\end{tabular}

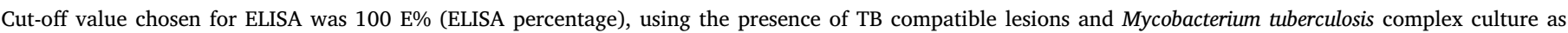
reference standards.

a $95 \%$ confidence intervals.
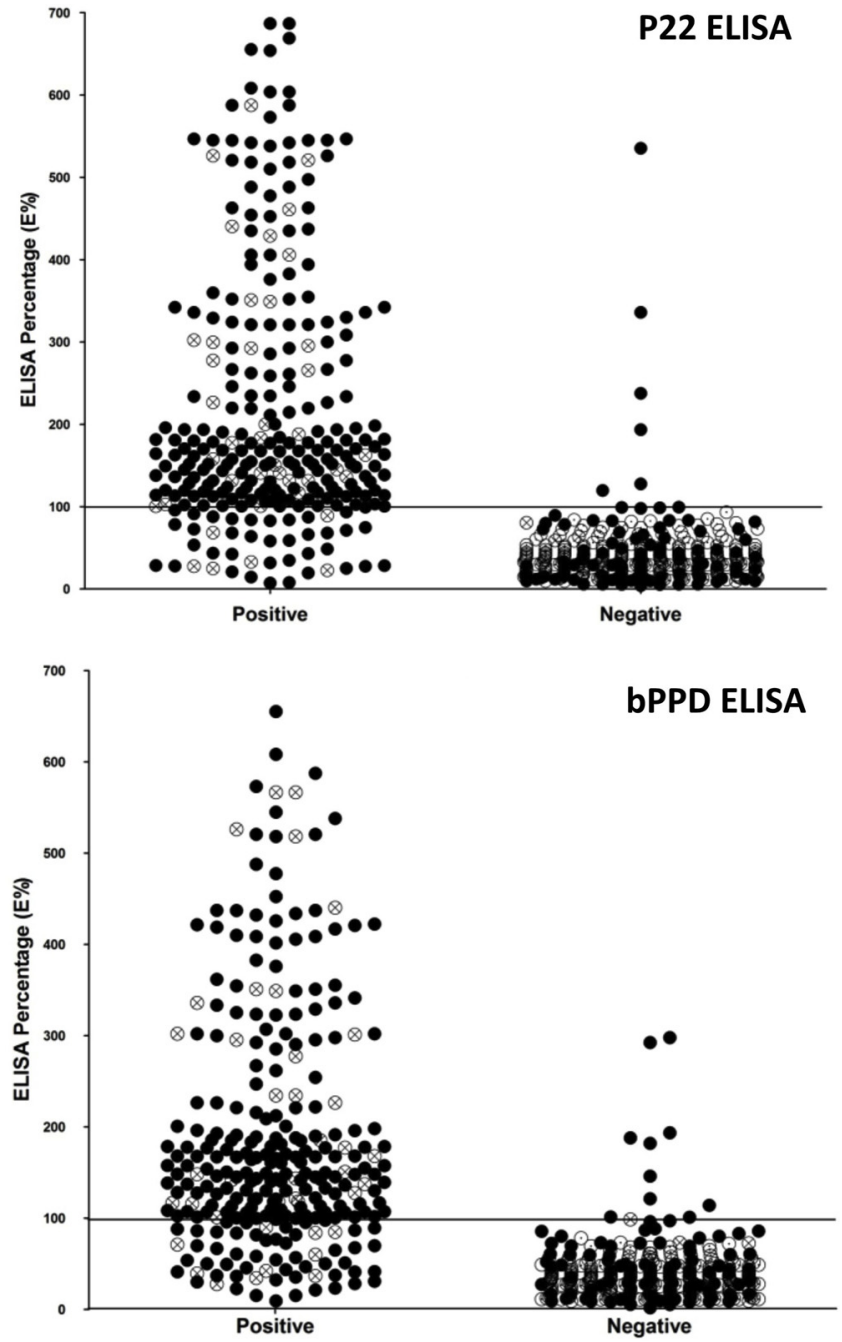

Fig. 2. Histogram of the distribution of the ELISA percentage (E\%) values of individual animals tested by ELISA using P22 and bPPD as antigens, respectively, according to their known TB status. (Wild boar - $\bullet$, Iberian pig - $\bigotimes$, White pig - $\odot$ ). The horizontal line represents the chosen cut-off value.

reared under intensive systems of management (white pigs), in both bPPD and P22 ELISA. The histogram of the species-wise distribution of the ELISA value (E\%) of individual TB positive and negative animals is represented in Fig. 2. Positive and negative predictive values are found to be higher in P22 ELISA than in bPPD (Table 3), which indicates comparatively higher diagnostic accuracy for P22 ELISA and a higher proportion of number of cases identified out of all positive test results.

\section{Discussion}

This study confirms that serological tests are valuable tools in the diagnosis of TB in wild boar and pigs. Moreover, the new P22 ELISA yielded a high serodiagnostic value in terms of Sp (98.4\%) and Se (84.1\%), improving the performance of the classic bPPD ELISA (Sp: $97.3 \%$, Se: $77.3 \%)$.

Antibody diagnostic tests can be routinely used in the diagnosis and surveillance of TB in swine, as they constitute a rapid and cost-effective screening tool for samples that can be collected both ante and post mortem. Our study reinforces previous findings stating that these tests have a high diagnostic value (Table 1). In addition, antibody based diagnosis has been found to be more reliable in swine when compared with other species. Antibody production is closely related to infection status/lesion severity in swine, as found in several studies (Aurtenetxe et al., 2008; Lyashchenko et al., 2008; Garrido et al., 2011; Roos et al., 2016; Cardoso-Toset et al., 2017). In contrast, this correlation is not consistently reported in ruminants such as red deer (Cervus elaphus) (Thomas et al., 2017), fallow deer (Dama dama) (Boadella et al., 2012), cattle (Bezos et al., 2014) or goats (Gutiérrez et al., 1998). This is also evidenced by the higher sum of Se $+\mathrm{Sp}$ in the ROC analysis, along with a high kappa agreement with the reference standards when compared to studies carried out on other species (Boadella et al., 2012; Buendía et al., 2013; Kang et al., 2016). The results obtained with the new antigen P22 coincide with recent reports of a high Sp and Se in mice (Infantes-Lorenzo et al., 2017) and a higher Se in cattle (Casal et al., 2017) when compared to bPPD.

The high $\mathrm{Sp}$ is attributed to the fact that the P22 antigen shares fewer proteins than bPPD with other mycobacteria not belonging to the MTC (Infantes-Lorenzo et al., 2017). This may minimize the proportion of false positive reactions in non-infected animals (Morés et al., 2006). The Sp of the bPPD ELISA was $100 \%$ in some previous studies (see Table 1), but either the sample size used for those studies was very low (experiments), or negative samples were taken from TB free herds. In this context, our study is innovative, since it includes a large sample size and field samples from wild boar and domestic pigs, regardless of their origin. A strict comparison with other previous studies is, therefore, difficult. However, the high Sp found in the aforementioned studies coincides with the finding we obtained in the case of white pigs in the present study, in which the Sp was $100 \%$ for both ELISAs. These pigs, which are housed in closed farm buildings, are not having exposure to mycobacteria able to elicit a cross reactive immunity that would influence on test Sp (Estevez et al., 2004). This contrasts with the possibility of exposure in the case of free ranging Iberian pigs and wild boar. Even so, the high global Sp of P22 ELISA (98.4\%) observed in this study confirms that it could be used in epidemiological studies focused on farms as the unit of study, where the identification of a seropositive animal indicates with high probability that a herd is truly infected.

A good Se was found in both ELISAs, which is in line with previous findings reporting that $50 \%$ of the concentration of the 10 principal proteins of $M$. bovis is found in both bPPD and P22, five of which are predicted to be highly immunogenic (Infantes-Lorenzo et al., 2017). 
The higher Se achieved in the P22 ELISA in this study confirms the hypothesis that suggests that replacing bPPD with P22 may increase the Se of the immunological diagnosis of TB because of the high abundance of MPB70 in M. bovis and its immunological properties (Harboe et al., 1990; Infantes-Lorenzo et al., 2017). Similarly, in the study of GarcíaBocanegra et al. (2012), a better Se was obtained with the MPB83 antigen in comparison to bPPD showing that, in addition to the high Sp, it is also possible to have a better Se with purified/specific antigens than with the whole bPPD protein complex.

This study has also limitations for the interpretation of the results. We used presence or absence of TBL as well as MTC culture as reference tests in order to determine the TB status of the animals. This means that the Se might be overestimated, due to the definition of a positive TB status in this study that causes a bias towards advanced cases of TB and its consequence on overall test performance, since the antibody response is more active towards the later stage of the disease (Waters et al., 2016). Moreover, it could be possible to find some infected animals without visible TBLs or false negative results in culture due to the possibility of the presence of microorganisms in the non-cultured tissues or organs which had no visible TBLs or due to decontamination prior to MTC culture that adversely affects the viability of mycobacteria, especially when the number of viable microorganisms is low (Stewart et al., 2013). Mycobacterial culture is often considered as an imperfect gold standard because if the gold standard test itself does not have a $100 \%$ Se, errors will arise in defining the Se of new diagnostic assays under validation (Banoo et al., 2008; Maas et al., 2013). Hence, in some cases requiring improved accuracy, the P22 ELISA could be interpreted in parallel to the diagnostic techniques based on CMI response, in order to improve the Se, as reported in other species (Harrington et al., 2008; Casal et al., 2017).

\section{Conclusion}

In conclusion, the serodiagnosis of TB in suids (pig and wild boar) is an efficient and simple method by which to monitor farmed or wild animal populations for the purpose of TB prevention and control. The immunopurified antigen complex P22 provided a better Se and Sp when compared to bPPD when used in an indirect ELISA for MTC-antibody detection. The P22-based indirect ELISA can be used as a cost effective, rapid and reliable tool for the large-scale screening and assessment of the TB status of wild boar and pigs.

\section{Conflict of interests}

The authors declare no conflicts of interest.

\section{Acknowledgements}

Research funding was provided by 'Plan Nacional' grant WildDriver CGL2017-89866 (MINECO, Spain and EU FEDER), the 3GdiagnoTB project (RTC-2016-4746-2, MINECO, Spain), and TAVS-CM Programme of the Comunidad de Madrid (S2013/ABI-2747). J. Thomas was supported by a grant from the Indian Council of Agricultural ResearchInternational Fellowship 2014-15 (ICAR-IF 2014-15). MA. Risalde holds a postdoctoral contract from the Research Plan of the University of Córdoba and the Operational Program of EU-FEDER funds from Andalucía. The authors would like to thank F. Talavera and other IREC and UCO staff for their technical assistance. We are also thankful to Sabiotec for providing valuable samples.

\section{Appendix A. Supplementary data}

Supplementary material related to this article can be found, in the online version, at doi:https://doi.org/10.1016/j.prevetmed.2018.11. 004.

\section{References}

Arenas-Montes, A., García-Bocanegra, I., Paniagua, J., Franco, J.J., Miró, F., FernándezMorente, M., Arenas, A., 2013. Blood sampling by puncture in the cavernous sinus from hunted wild boar. Eur. J. Wildlife Res. 59, 299-303.

Aurtenetxe, O., Barral, M., Vicente, J., de la Fuente, J., Gortázar, C., Juste, R.A., 2008. Development and validation of an enzyme-linked immunosorbent assay for antibodies against Mycobacterium bovis in European wild boar. BMC Vet. Res. 4, 43.

Bailey, S.S., Crawshaw, T.R., Smith, N.H., Palgrave, C.J., 2013. Mycobacterium bovis infection in domestic pigs in Great Britain. Vet. J. 198, 391-397.

Banoo, S., Bell, D., Bossuyt, P., Herring, A., Mabey, D., Poole, F., Smith, P.G., Sriram, N., Wongsrichanalai, C., Linke, R., O'brien, R., 2008. Evaluation of diagnostic tests for infectious diseases: general principles. Nat. Rev. Microbiol. 6, 16-28.

Beltrán-Beck, B., Romero, B., Boadella, M., Casal, C., Bezos, J., Mazariegos, M., Martín, M., Galindo, R.C., de la Lastra, J.M.P., Villar, M., Garrido, J.M., 2014. Tonsils of the soft palate do not mediate the response of pigs to oral vaccination with heat-inactivated Mycobacterium bovis. Clin. Vaccine Immunol. 21, 1128-1136.

Bezos, J., Casal, C., Romero, B., Schroeder, B., Hardegger, R., Raeber, A.J., López, L., Rueda, P., Domínguez, L., 2014. Current ante-mortem techniques for diagnosis of bovine tuberculosis. Res. Vet. Sci. 97, 44-S52.

Boadella, M., Barasona, J.A., Diaz-Sanchez, S., Lyashchenko, K.P., Greenwald, R., Esfandiari, J., Gortázar, C., 2012. Performance of immunochromatographic and ELISA tests for detecting fallow deer infected with Mycobacterium bovis. Prev. Vet. Med. 104, 60-164.

Boadella, M., Lyashchenko, K., Greenwald, R., Esfandiari, J., Jaroso, R., Carta, T., Garrido, J.M., Vicente, J., de la Fuente, J., Gortázar, C., 2011. Serologic tests for detecting antibodies against Mycobacterium bovis and Mycobacterium avium subspecies paratuberculosis in Eurasian wild boar (Sus scrofa scrofa). J. Vet. Diagn. Invest. 23, 77-83.

Buddle, B.M., Livingstone, P.G., De Lisle, G.W., 2009. Advances in ante-mortem diagnosis of tuberculosis in cattle. N. Z. Vet. J. 57, 173-180.

Buendía, A.J., Navarro, J.A., Salinas, J., McNair, J., de Juan, L., Ortega, N., Cámara, P., Torreblanca, P., Sanchez, J., 2013. Ante-mortem diagnosis of caprine tuberculosis in persistently infected herds: influence of lesion type on the sensitivity of diagnostic tests. Res. Vet. Sci. 95, 1107-1113.

Cardoso-Toset, F., Luque, I., Carrasco, L., Jurado-Martos, F., Risalde, M.Á, Venteo, Á., Infantes-Lorenzo, J.A., Bezos, J., Rueda, P., Tapia, I., Gortázar, C., 2017. Evaluation of five serologic assays for bovine tuberculosis surveillance in domestic free-range pigs from southern Spain. Prev. Vet. Med. 137, 101-104.

Casal, C., Infantes, J.A., Risalde, M.A., Díez-Guerrier, A., Domínguez, M., Moreno, I., Romero, B., de Juan, L., Sáez, J.L., Juste, R., Gortázar, C., 2017. Antibody detection tests improve the sensitivity of tuberculosis diagnosis in cattle. Res. Vet. Sci. 112, 214-221.

Chambers, M.A., 2013. Review of the diagnosis of tuberculosis in non-bovid wildlife species using immunological methods-an update of published work since 2009. Transbound. Emerg. Dis. 60, 14-27.

Che'Amat, A., González-Barrio, D., Ortiz, J.A., Díez-Delgado, I., Boadella, M., Barasona, J.A., Bezos, J., Romero, B., Armenteros, J.A., Lyashchenko, K.P., Venteo, A., 2015. Testing Eurasian wild boar piglets for serum antibodies against Mycobacterium bovis. Prev. Vet. Med. 121, 93-98.

Cohen, J.F., Korevaar, D.A., Altman, D.G., Bruns, D.E., Gatsonis, C.A., Hooft, L., Irwig, L., Levine, D., Reitsma, J.B., De Vet, H.C., Bossuyt, P.M., 2016. STARD 2015 guidelines for reporting diagnostic accuracy studies: explanation and elaboration. BMJ Open 6, e012799.

Coleman, J.D., Cooke, M.M., 2001. Mycobacterium bovis infection in wildlife in New Zealand. Tuberculosis 81, 191-202.

Corner, L.A., Trajstman, A.C., 1988. An evaluation of 1-hexadecylpyridinium chloride as a decontaminant in the primary isolation of Mycobacterium bovis from bovine lesions. Vet. Microbiol. 18, 127-134.

Corner, L.A., 2006. The role of wild animal populations in the epidemiology of tuberculosis in domestic animals: how to assess the risk. Vet. Microbiol. 112, 303-312.

Csivincsik, Á., Rónai, Z., Nagy, G., Svéda, G., Halász, T., 2016. Surveillance of Mycobacterium caprae infection in a wild boar (Sus scrofa) population in south-western Hungary. Vet. Arhiv. 86, 767-775.

De la Rua-Domenech, R., Goodchild, A.T., Vordermeier, H.M., Hewinson, R.G., Christiansen, K.H., Clifton-Hadley, R.S., 2006. Ante mortem diagnosis of tuberculosis in cattle: a review of the tuberculin tests, $\gamma$-interferon assay and other ancillary diagnostic techniques. Res. Vet. Sci. 81, 190-210.

Di Marco, V., Mazzone, P., Capucchio, M.T., Boniotti, M.B., Aronica, V., Russo, M., Fiasconaro, M., Cifani, N., Corneli, S., Biasibetti, E., Biagetti, M., 2012. Epidemiological significance of the domestic black pig (Sus scrofa) in maintenance of bovine tuberculosis in Sicily. J. Clin. Microbiol. 50, 1209-1218.

El Mrini, M., Kichou, F., Kadiri, A., Berrada, J., Bouslikhane, M., Cordonnier, N., Romero, B., Gortázar, C., 2016. Animal tuberculosis due to Mycobacterium bovis in Eurasian wild boar from Morocco. Eur. J. Widl. Res 62, 479-482.

Estévez, M., Morcuende, D., Ramirez, R., Ventanas, J., Cava, R., 2004. Extensively reared Iberian pigs versus intensively reared white pigs for the manufacture of liver pâté. Meat Sci. 67, 453-461.

García-Bocanegra, I., de Val, B.P., Arenas-Montes, A., Paniagua, J., Boadella, M., Gortázar, C., Arenas, A., 2012. Seroprevalence and risk factors associated to Mycobacterium bovis in wild artiodactyl species from southern Spain, 2006-2010. PLoS One 7, e34908.

Garrido, J.M., Sevilla, I.A., Beltrán-Beck, B., Minguijón, E., Ballesteros, C., Galindo, R.C., Boadella, M., Lyashchenko, K.P., Romero, B., Geijo, M.V., Ruiz-Fons, F., 2011. Protection against tuberculosis in Eurasian wild boar vaccinated with heat- 
inactivated Mycobacterium bovis. PLoS One 6, e24905.

Gortázar, C., Che Amat, A., O'brien, D.J., 2015. Open questions and recent advances in the control of a multi-host infectious disease: animal tuberculosis. Mammal Rev. 45, $160-175$.

Gortázar, C., Delahay, R.J., Mcdonald, R.A., Boadella, M., Wilson, G.J., Gavier-Widen, D., Acevedo, P., 2012. The status of tuberculosis in European wild mammals. Mammal Rev. 42, 193-206

Gortázar, C., Fernández-Calle, L.M., Collazos-Martínez, J.A., Mínguez-González, O., Acevedo, P., 2017. Animal tuberculosis maintenance at low abundance of suitable wildlife reservoir hosts: a case study in northern Spain. Prev. Vet. Med. 146, 150-157.

Gutiérrez, M., Tellechea, J., Marín, J.F.G., 1998. Evaluation of cellular and serological diagnostic tests for the detection of Mycobacterium bovis-infected goats. Vet. Microbiol. 62, 281-290.

Harboe, M., Wiker, H.G., Duncan, J.R., Garcia, M.M., Dukes, T.W., Brooks, B.W., Turcotte, C., Nagai, S., 1990. Protein G-based enzyme-linked immunosorbent assay for antiMPB70 antibodies in bovine tuberculosis. J. Clin. Microbiol. 28, 913-921.

Harrington, N.P., Surujballi, O.P., Prescott, J.F., Duncan, J.R., Waters, W.R., Lyashchenko, K., Greenwald, R., 2008. Antibody responses of cervids (Cervus elaphus) following experimental Mycobacterium bovis infection and the implications for immunodiagnosis. Clin. Vaccine Immunol. 15, 1650-1658.

Hlokwe, T.M., Van Helden, P., Michel, A.L., 2014. Evidence of increasing intra and interspecies transmission of Mycobacterium bovis in South Africa: are we losing the battle? Prev. Vet. Med. 115, 10-17.

Infantes-Lorenzo, J.A., Moreno, I., de los Ángeles Risalde, M., Roy, Á., Villar, M., Romero, B., Ibarrola, N., de la Fuente, J., Puentes, E., de Juan, L., Gortázar, C., 2017. Proteomic characterisation of bovine and avian purified protein derivatives and identification of specific antigens for serodiagnosis of bovine tuberculosis. Clin. Proteom. Bioinform. 14, 36

Jang, Y., Ryoo, S., Lee, H., Kim, N., Lee, H., Park, S.Y., Song, W.S., Kim, J.T., Lee, H.S., Myung Kim, J., 2017. Isolation of Mycobacterium bovis from free-ranging wildlife in South Korea. J. Wildl. Dis. 53, 181-185.

Jaroso, R., Vicente, J., Fernandez-de-Mera, I.G., Aranaz, A., Gortázar, C., 2010. Eurasian wild boar response to skin-testing with mycobacterial and non-mycobacterial antigens. Prev. Vet. Med. 96, 211-217.

Kamerbeek, J., Schouls, L.E.O., Kolk, A., Van Agterveld, M., Van Soolingen, D., Kuijper, S., Bunschoten, A., Molhuizen, H., Shaw, R., Goyal, M., Van Embden, J., 1997. Simultaneous detection and strain differentiation of Mycobacterium tuberculosis for diagnosis and epidemiology. J. Clin. Microbiol. 35, 907-914.

Kang, S.S., Byeon, H.S., Ku, B.K., Kim, S.W., Kim, J., Woo, J., Ahn, B., Kim, S., Monoldorova, S., Park, C.H., Cho, S.N., 2016. Seroprevalence of tuberculosis in domesticated elk (Cervus canadensis) in Korea. Res. Vet. Sci. 107, 228-232.

Lyashchenko, K.P., Greenwald, R., Esfandiari, J., Chambers, M.A., Vicente, J., Gortazar, C., Santos, N., Correia-Neves, M., Buddle, B.M., Jackson, R., O’Brien, D.J., 2008. Animal-side serologic assay for rapid detection of Mycobacterium bovis infection in multiple species of free-ranging wildlife. Vet. Microbiol. 132, 283-292.

Maas, M., Michel, A.L., Rutten, V.P., 2013. Facts and dilemmas in diagnosis of tuberculosis in wildlife. Comp. Immunol. Microbiol. Infect. Dis. 36, 269-285.

Maciel, A.L.G., Loiko, M.R., Bueno, T.S., Moreira, J.G., Coppola, M., Dalla Costa, E.R., Schmid, K.B., Rodrigues, R.O., Cibulski, S.P., Bertagnolli, A.C., Mayer, F.Q., 2018. Tuberculosis in Southern Brazilian wild boars (Sus scrofa): First epidemiological findings. Transbound. Emerg. Dis. 65, 518-526.

Mayer, F.Q., Cerva, C., Driemeier, D., da Cruz, C.E.F., Loiko, M.R., de Menezes Coppola, M., Cibulski, S., Bertagnolli, A.C., 2012. Mycobacterium bovis infection in a collared peccary (Tayassu tajacu): insights on tuberculosis wild reservoirs. Vet. Microbiol. 160,
$549-551$.

Michel, A.L., Müller, B., van Helden, P.D., 2010. Mycobacterium bovis at the animal-human interface: a problem, or not? Vet. Microbiol. 140, 371-381.

Miller, M., Buss, P., de Klerk-Lorist, L.M., Hofmeyr, J., Hausler, G., Lyashchenko, K., Lane, E.P., Botha, L., Parsons, S., van Helden, P., 2016. Application of rapid serologic tests for detection of Mycobacterium bovis infection in free-ranging warthogs (Phacochoerus africanus)-Implications for antemortem disease screening. J. Wildl. Dis. 52, 180-182.

Morés, N., Amaral, A.L., Ventura, L., Silva, R.A.M., Silva, V.S., Barioni Junior, W., 2006 Comparative study of methods of tuberculin skin test with the avium and bovine tuberculin in the diagnosis of the infection by agents of the Mycobacterium aviumcomplex or M. Bovis in swine. Arq. Bras. Med. Vet. Zoo. 58, 708-717.

Mohamed, A.M., El-Ella, G.A.A., Nasr, E.A., Soliman, Y.A., 2012. Evaluation of fibronectin-binding protein Ag85-B as target for serodiagnosis of swine mycobacteriosis in living animals. J. Mycobac. Dis. S. 2 2161-1068.

Naranjo, V., Gortázar, C., Vicente, J., De La Fuente, J., 2008. Evidence of the role of European wild boar as a reservoir of Mycobacterium tuberculosis complex. Vet. Microbiol. 127, 1-9.

Nugent, G., Gortázar, C., Knowles, G., 2015. The epidemiology of Mycobacterium bovis in wild deer and feral pigs and their roles in the establishment and spread of bovine tuberculosis in New Zealand wildlife. N. Z. Vet. J. 63, 54-67.

Pedersen, K., Miller, R.S., Anderson, T.D., Pabilonia, K.L., Lewis, J.R., Mihalco, R.L., Gortázar, C., Gidlewski, T., 2017. Limited antibody evidence of exposure to Mycobacterium bovis in feral swine (Sus scrofa) in the USA. J. Wildl. Dis. 53, 30-36.

Pérez de Val, B., Napp, S., Velarde, R., Lavín, S., Cervera, Z., Singh, M., Allepuz, A., Mentaberre, G., 2017. Serological follow-up of tuberculosis in a wild boar population in contact with infected cattle. Transbound. Emerg. Dis. 64, 275-283.

Pesciaroli, M., Russo, M., Mazzone, P., Aronica, V., Fiasconaro, M., Boniotti, M.B. Corneli, S., Cagiola, M., Pacciarini, M., Di Marco, V., Pasquali, P., 2012. Evaluation of the interferon-gamma (IFN- $\gamma$ ) assay to diagnose Mycobacterium bovis infection in pigs. Vet. Immunol. Immunopathol. 148, 369-372.

Richomme, C., Boschiroli, M.L., Hars, J., Casabianca, F., Ducrot, C., 2010. Bovine tuberculosis in livestock and wild boar on the Mediterranean Island, Corsica. J. Wildl. Dis. $46,627-631$.

Roos, E.O., Buss, P., de Klerk-Lorist, L.M., Hewlett, J., Hausler, G.A., Rossouw, L., McCall, A.J., Cooper, D., van Helden, P.D., Parsons, S.D., Miller, M.A., 2016. Test performance of three serological assays for the detection of Mycobacterium bovis infection in common warthogs (Phacochoerus africanus). Vet. Immunol. Immunopathol. 182, 79-84.

Roos, E.O., Olea-Popelka, F., Buss, P., de Klerk-Lorist, L.M., Cooper, D., van Helden, P.D., Parsons, S.D.C., Miller, M.A., 2018. Seroprevalence of Mycobacterium bovis infection in warthogs (Phacochoerus africanus) in bovine tuberculosis-endemic regions of South Africa. Transbound. Emerg. Dis. 65, 1182-1189.

Stewart, L.D., McNair, J., McCallan, L., Gordon, A., Grant, I.R., 2013. Improved detection of Mycobacterium bovis infection in bovine lymph node tissue using immunomagnetic separation (IMS)-based methods. PLoS One 8, e58374.

Thomas, J., Risalde, M.Á., Serrano, M., Sevilla, I., Geijo, M., Ortíz, J.A., Fuertes, M., RuízFons, J.F., de la Fuente, J., Domínguez, L., Juste, R., 2017. The response of red deer to oral administration of heat-inactivated Mycobacterium bovis and challenge with a field strain. Vet. Microbiol. 208, 195-202.

Waters, W.R., Maggioli, M.F., Palmer, M.V., Thacker, T.C., McGill, J.L., Vordermeier, H.M., Berney-Meyer, L., Jacobs, W.R., Larsen, M.H., 2016. Interleukin-17A as a biomarker for bovine tuberculosis. Clin. Vaccine Immunol. 23, 168-180.

Wilton, S., Cousins, D., 1992. Detection and identification of multiple mycobacterial pathogens by DNA amplification in a single tube. Genome Res. 1, 69-273. 